\title{
Computational Study of Second- and Third-Harmonic Generation in Periodically Patterned 2D-3D Heteromaterials
}

\author{
Martin Weismann ${ }^{1}$ and Nicolae C. Panoiu ${ }^{1}$
}

\begin{abstract}
Remarkable optical and electrical properties of graphene and other two-dimensional (2D) materials provide significant potential for novel optoelectronic applications and devices, many of which depend on nonlinear optical effects in these 2D materials. In this paper we use a theoretical and computational formalism we have recently introduced to efficiently and accurately compute the linear and nonlinear optical response of nanostructured 2D materials embedded in periodic structures containing regular three-dimensional (3D) materials, such as diffraction gratings or periodic metamaterials. Thus, we use the proposed method to demonstrate enhanced nonlinear optical interactions in periodically patterned photonic nanostructures via resonant excitation of phase-matched nonlinear waveguide modes, enhanced nonlinearity of nanostructures containing graphene and other 2D nanomaterials, such as $\mathrm{WS}_{2}$, and multi-continua Fano resonances for increasing the nonlinear efficiency of hybrid 2D-3D photonic heteromaterials.
\end{abstract}

\section{INTRODUCTION}

Since its first isolation, preparation, and theoretical description [1], graphene, a monolayer of carbon atoms in a hexagonal lattice, has attracted a tremendous amount of interest in science and engineering due to its outstanding physical properties and potential for novel applications. Graphene was shown to have remarkable mechanic strength at a low density [2], [3], [4] and exhibit high thermal conductivity [5], making it particularly relevant to nano-electromechanical applications and thermal management in nano-electric circuits [3], [6]. In addition, the high carrier mobility of graphene open up the potential for high-frequency electronic applications [7], [8]. These remarkable properties of an atomically thin monolayer of graphite spurred the development of new two-dimensional (2D) materials, such as hexagonal boron nitride $(h-\mathrm{BN})$, silicene (monolayers of silicon), and transition metal dichalcogenide (TMDC) monolayers [9], [10], each with their own sets of unusual physical properties.

One additional compelling aspect are the optical properties of 2D materials (2DM). Graphene, for example, is nearly transparent at optical frequencies, exhibiting absorption of 2.3\% of white light [11]. This transparency and the just mentioned electro-mechanical properties make graphene a promising new material for flexible optical devices, e.g. touch screens [12]. In addition, nonlinear optical properties of graphene, namely third harmonic generation (THG), a

\footnotetext{
This work was supported by the European Research Council / ERC Grant Agreement no. ERC-2014-CoG-648328

${ }^{1}$ The authors are with Department of Electronic and Electrical Engineering, University College London, Torrington Place, London WC1E 7JE, UK martin.weismann.12@ucl.ac.uk; n.panoiu@ucl.ac.uk
}

strong optical Kerr nonlinearity, and induced second order nonlinearity, will allow applications of graphene to ultracompact modulators, optical limiters, frequency converters, and to photovoltaic and photoresistive devices [12]. Similar possibilities are envisioned for other 2D materials, e.g. for the semiconducting TMDC monolayers [13]. The implementation of these optical properties into applications requires advances of fabrication and experimental techniques [12], [14], theoretical models, and, equally important, numerical methods for modeling of devices incorporating 2D materials.

In this paper, we present a numerical method for nanopatterned 2D-3D photonic structures and use it to demonstrate enhanced nonlinear optical interactions in periodically patterned photonic nanostructures via resonant excitation of phase-matched nonlinear waveguide modes, enhanced nonlinearity of nanostructures containing graphene and other 2D nanomaterials, such as $\mathrm{WS}_{2}$, and multicontinua Fano resonances for increasing the nonlinear efficiency of hybrid 2D-3D photonic heteromaterials.

\section{Computational Approach Describing HARMONIC GENERATION IN 2D MATERIALS}

Our numerical method [15] for 2D-3D heterostructures is based on a general physical description of the linear and nonlinear optical properties of $2 \mathrm{D}$ materials and on the rigorous coupled-wave analysis (RCWA).

\section{A. Linear and nonlinear optical properties of $2 D$ materials}

In principle, the interaction of light with sheets of 2D materials can be described by conventional numerical methods in computational electromagnetics, e.g. FDTD [16] or FEM [17], by defining and effective thickness of the 2D material and incorporating it into the computational algorithm simply as a very thin layer of 3D material [18]. This can however lead to numerical artefacts due to a size-imbalance between the characteristic length of the photonic structure and the small thickness of the 2D material, which is by itself an ambiguous quantity, and is computationally inefficient. We follow a more suitable approach by incorporating the 2D material into the algorithm as a conductive sheet, which gives rise to a surface current density [19]

$$
\mathbf{j}=\sigma^{(1)} \mathbf{E}+\mathbf{j}^{\mathrm{nl}}(\mathbf{E})
$$

Hereby, $\mathbf{E}$ denotes the complex amplitude of the electric field at a given frequency $\omega, \sigma^{(1)}$ is the linear sheet conductance of the $2 \mathrm{D}$ material and $\mathbf{j}^{\mathrm{nl}}$ is the nonlinear surface current, which depends nonlinearly on $\mathbf{E}$. 
In the case of graphene, $\sigma^{(1)}$ can be written as [20]

$$
\begin{aligned}
\frac{\sigma^{(1)}(\omega)}{\sigma_{0}}= & \frac{4 \varepsilon_{F}}{\pi \hbar} \frac{i}{\omega+i \tau^{-1}} \\
& +\theta\left(\hbar \omega-2 \varepsilon_{F}\right)+\frac{i}{\pi} \ln \left|\frac{\hbar \omega-2 \varepsilon_{F}}{\hbar \omega+2 \varepsilon_{F}}\right|,
\end{aligned}
$$

where $\sigma_{0}=e^{2} /(4 \hbar)=6.089 \times 10^{-5} \mathrm{~A} \mathrm{~V}^{-1}$ is the universal dynamic conductivity of graphene, $e$ denotes the elementary charge, $\varepsilon_{F}=0.6 \mathrm{eV}$ is the Fermi level used in our study, $\hbar$ the reduced Planck constant, $\tau=0.25 \mathrm{ps}$ the relaxation time and $\theta(\cdot)$ is the Heaviside step function. The nonlinear properties of $2 \mathrm{D}$ materials depend on their symmetry properties. Graphene is centrosymmetric and therefore even-order nonlinear processes, such as second harmonic generation (SHG), are forbidden. Instead, graphene exhibits strong thirdharmonic generation (THG) [21], [22] which is described by $\mathbf{j}^{\mathrm{nl}}(\mathbf{E})=\sigma^{(3)} \mathbf{E}|\mathbf{E} \cdot \mathbf{E}|$. The third order conductance, $\sigma^{(3)}$, is given by [22]

$$
\sigma^{(3)}(\omega)=\frac{i \sigma_{0}\left(\hbar v_{F} e\right)^{2}}{48 \pi(\hbar \omega)^{4}} T\left(\frac{\hbar \omega}{2 \varepsilon_{F}}\right)
$$

Hereby, $T(x)=17 G(x)-64 G(2 x)+45 G(3 x)$ and $G(x)=$ $\ln \left|\frac{1+x}{1-x}\right|+i \pi \theta(|x|-1)$ with the Fermi velocity $v_{F}=$ $3 a_{0} \gamma_{0} /(2 \hbar) \approx c / 300$, the nearest-neighbor distance between the carbon atoms $a_{0}=1.42 \AA$, and the nearest-neighbor coupling constant $\gamma_{0}=2.7 \mathrm{eV}$.

\section{B. The rigorous coupled-wave analysis with inhomogeneous $S$-matrix formalism}

The RCWA [23], [24] is a modal frequency domain method for modeling light diffraction by 2D periodic structures, such as the generic multilayer grating shown in Fig. 1. In addition to nano-structured 3D materials, we show how to model periodically patterned $2 \mathrm{D}$ material distributions. In the RCWA, the electromagnetic (EM) field in each computational layer is described by its modes, i.e. its EM eigensolutions, and each mode is given as a Fourier series, which naturally incorporates the pseudo-periodicity of the EM solution. The Fourier series coefficients of the electric $\left(\left[E_{\mathbf{t}}^{(A)}\right]\right.$ and $\left.\left[E_{\mathbf{t}}^{(B)}\right]\right)$ and magnetic fields $\left(\left[H_{\mathbf{t}}^{(A)}\right]\right.$ and $\left.\left[H_{\mathbf{t}}^{(B)}\right]\right)$ in the two layers $A$ and $B$ adjacent to a 2D material sheet have to be determined by fulfilling the electromagnetic boundary conditions

$$
\begin{aligned}
& {\left[E_{\mathbf{t}}^{(A)}\left(z_{s}\right)\right]-\left[E_{\mathbf{t}}^{(B)}\left(z_{s}\right)\right]=0,} \\
& {\left[H_{\mathbf{t}}^{(A)}\left(z_{s}\right)\right]-\left[H_{\mathbf{t}}^{(B)}\left(z_{s}\right)\right]=\left[\delta H_{\mathbf{t}}^{(s)}\right] .}
\end{aligned}
$$

Hereby, the subscript $\mathbf{t}$ denotes the tangential components of a vector, $[f]$ denotes the vector of Fourier series coefficients of a function $f$ and $\delta H_{\mathbf{t}}^{(s)}=\left(j_{y},-j_{x}\right)^{T}$ denotes the variation of the tangential components of the magnetic field, $\mathbf{H}$, across the 2D material sheet, which is given by the linear and nonlinear surface currents from (1).

The nonlinearity of the surface current is resolved by the undepleted pump approximation in a three-step computational algorithm: In the first step, the diffracted EM fields at the fundamental frequency (FF) are calculated by RCWA and

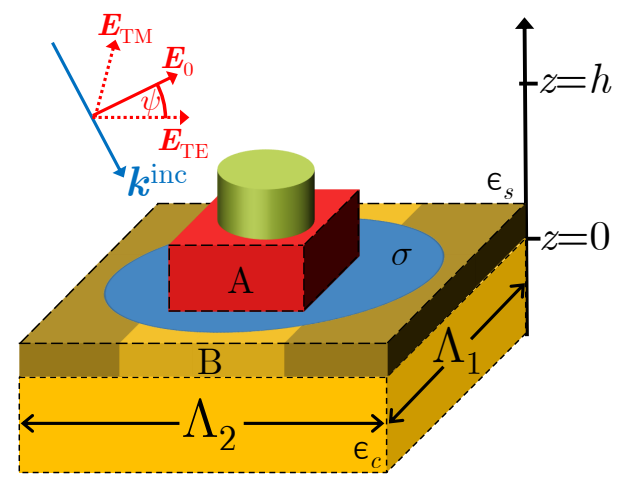

Fig. 1. Schematic of the unit cell of a generic multilayered 2D-periodic structure under plane wave incidence. Different computational RCWA layers made of structured 3D materials are encoded by colors and an additional 2D material layer is indicated by the blue sheet.

the boundary condition (5) with incorporation of the linear surface current $\mathbf{j}=\sigma^{(1)} \mathbf{E}$ but without the nonlinear surface current $\mathbf{j}^{\mathrm{nl}}$. The second step explicitly calculates $\mathbf{j}^{\mathrm{nl}}$ using the total diffracted field $\mathbf{E}$ at the FF. In the third step, the nonlinear source current $\mathbf{j}^{\mathrm{nl}}$ is incorporated into (5) by means of a numerically stable, inhomogeneous S-matrix algorithm, which facilitates the calculation of the nonlinear near- and far-field at the generated frequency.

Note that arbitrary 2D materials can be described in the formulation of (1) and (5) without assuming an effective thickness as long as their linear and nonlinear conductances are known. This widens the range of devices that can be modeled by our method.

\section{Surface Plasmons in Graphene Gratings}

As a first application, we investigate the resonant excitation of surface plasmons in a periodic array of graphene discs on a glass substrate. The diameter of the disks is $175 \mathrm{~nm}$ and the period is $\Lambda_{1}=\Lambda_{2}=250 \mathrm{~nm}$. We calculate the linear and nonlinear interaction of the graphene discs with normally incident light, linearly polarized light with wavelengths ranging from $2.5 \mu \mathrm{m}$ to $15 \mu \mathrm{m}$. The relative absorption of light in the graphene discs is depicted in Fig. 2(a). The generally increasing absorption towards larger wavelengths, is overlaid by distinct local maxima of absorption due to the excitation of surface plasmons around the graphene discs. For example, the electric near-field at $\lambda=5.02 \mu \mathrm{m}$ exhibits three maxima of strong local field enhancement around the graphene disc, as per Fig. 2(b). This field enhancement leads to increased THG, which is revealed by the nonlinear near-field profile for $\lambda_{\mathrm{TH}}=5.02 \mu \mathrm{m} / 3$ in Fig. 2(c), and by the radiation spectrum at the $\mathrm{TH}$. The dispersion of $\mathrm{TH}$ radiation follows the trend of the absorption spectrum and exhibits strongest radiated TH due to surface plasmon excitation.

\section{ENHANCED NONLINEARITY OF TMDC MONOLAYERS BY EXCITATION OF WAVEGUIDE MODES}

A different class of 2D materials are the semiconducting transition metal dichalcogenide (TMDC) monolayer materials, which are in contrast to graphene non-centrosymmetric 

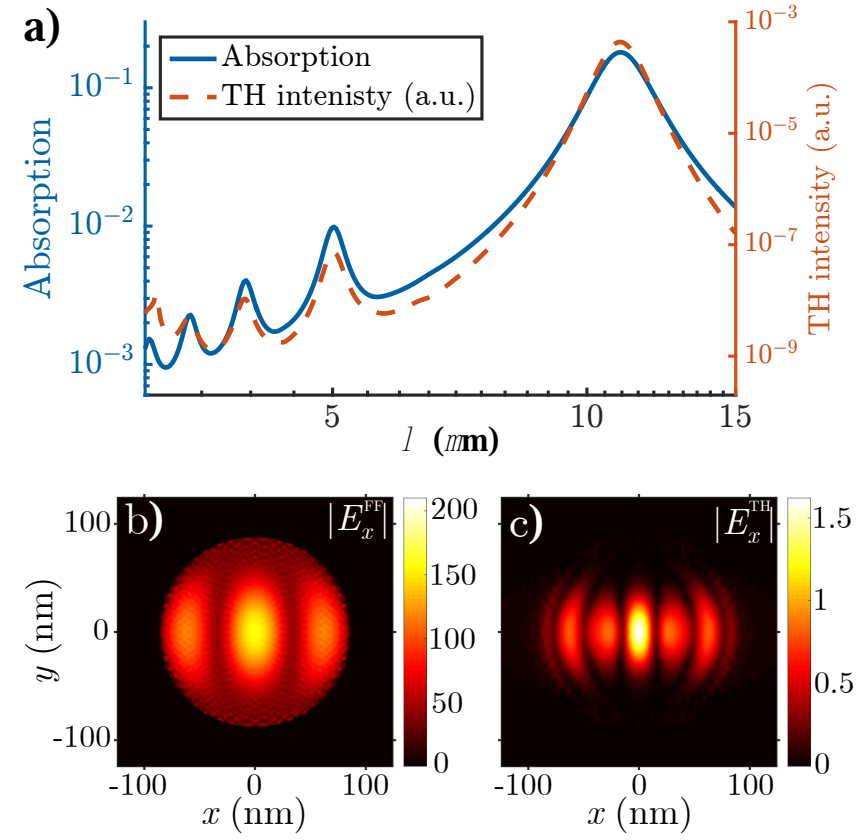

Fig. 2. a) Linear absorption and nonlinear radiation spectrum of graphene discs. b,c) Electric near-field distribution around graphene discs at FF (b) and $\mathrm{TH}(\mathrm{c})$.

and hence allow SHG. Here, we focus on a particular TMDC, $\mathrm{WS}_{2}$, for which the linear and nonlinear optical properties have recently been determined [25], [26]. Their quadratic optical nonlinearity is incorporated into the algorithm via the nonlinear surface current

$$
j_{\alpha}^{\mathrm{nl}}=\sum_{\beta, \gamma=x, y, z} \sigma_{\alpha \beta \gamma}^{(2)} E_{\beta} E_{\gamma}, \quad \alpha=x, y, z,
$$

where the nonlinear sheet conductance is taken from [26].

In contrast to the conductive graphene, $\mathrm{WS}_{2}$ does not support intrinsic resonances: because $\mathrm{WS}_{2}$ is semiconductor, it does not support plasmon resonances, and due to its vanishing thickness as a 2D material, no Mie-type resonances can be excited in any of the TMDCs. A different means of resonant excitation is investigated now, by combining a monolayer of $\mathrm{WS}_{2}$ with a periodically patterned slab waveguide as per in Fig. 3 (left panel). Such a slab waveguide supports optical modes and their excitation wavelength depends on the EM and geometric properties, most notably the height, $h$, of the waveguide. Thus, let the combined waveguide2D material device be illuminated by normally incident,

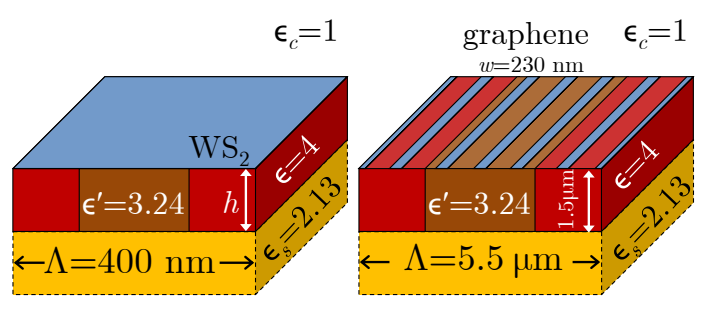

Fig. 3. Periodically patterned slab waveguides covered by a homogeneous $\mathrm{WS}_{2}$-monolayer (left) or graphene ribbons of width $w$ (right).

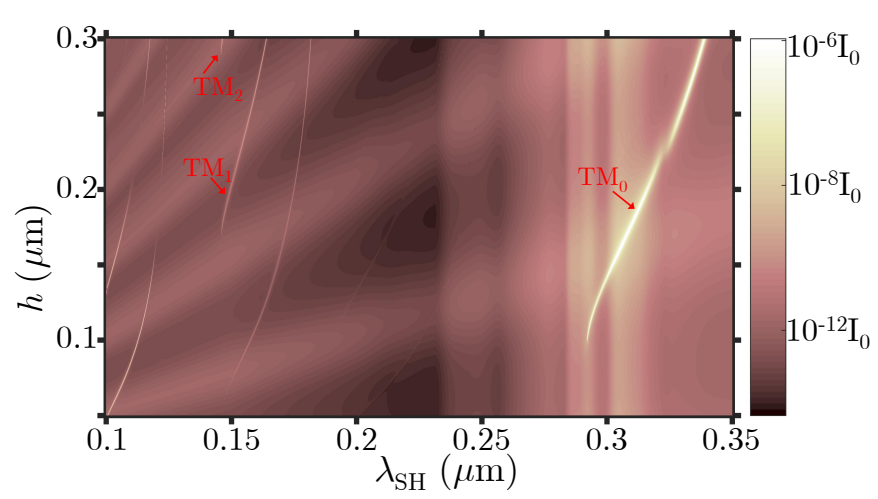

Fig. 4. Spectrum of SH radiation by a periodically patterned slab waveguide of height $h$ covered by a $\mathrm{WS}_{2}$ monolayer.

$x$-polarized light with wavelength ranging from $0.2 \mu \mathrm{m}$ to $0.7 \mu \mathrm{m}$ and with intensity of $I_{0}=1 \times 10^{12} \mathrm{~W} \mathrm{~m}^{-2}$. The intensity of radiated $\mathrm{SH}$ is depicted in Fig. 4 for a range of different heights of the waveguide, and it shows a mostly smooth variation of reflectivity with $\lambda$ and $h$ over different orders of magnitude due to Farby-Perot resonance at the FF and due to the intrinsic dispersion of $\sigma^{(2)}$. The strongest enhancement of $\mathrm{SH}$ is, however, du to the spectrally narrow excitation of the $\mathrm{TM}_{0}$ waveguide mode at the $\mathrm{FF}$, which yields the strongest enhancement of the fundamental field. This in turn generates the strongest nonlinear source current and therefore the highest intensity of radiated $\mathrm{SH}$ light.

\section{Multi-COntinua Fano Resonances in a Graphene-COVERED WAVEGUIDE}

As a last example we investigate a variation of the above introduced slab waveguide, where the monolayer of $\mathrm{WS}_{2}$ is replaced by resonant graphene ribbons, as per Fig. 3 (right panel). Its geometric parameters are designed such that the excitation wavelength of the fundamental $\mathrm{TM}_{0}$ waveguide mode is in the range of wavelengths at which surface plasmons are excited in the graphene ribbons. This is illustrated in Fig. 5: Similar to the absorption by graphene disks in Fig. 2(a), the linear absorption of the graphene ribbons increases for increasing wavelengths. It exhibits additional local absorption maxima due to the excitation of surface plasmons in the inner graphene ribbons on top of the waveguide material with permittivity $\epsilon^{\prime}=3.24$, e.g. at $\lambda=7.1 \mu \mathrm{m}$ in Fig. 6(a), or on top of the waveguide material with permittivity $\epsilon=4$, e.g. at $\lambda=7.6 \mu \mathrm{m}$ in Fig. 6(b). This field enhancement due to plasmon excitation also increases the intensity of radiated $\mathrm{TH}$, as per Fig. 5. The strongest $\mathrm{TH}$ radiation with intensity of $I_{\mathrm{TH}}=1 \times 10^{-3} I_{0}$ is obtained, however, due to the excitation of the $\mathrm{TM}_{0}$ waveguide mode at $\lambda=8.05 \mu \mathrm{m}$, for which the fundamental near-field is depicted in Fig. 6(c). This discrete waveguide resonance on the background of two spectrally broad plasmon resonances amounts to a double-continuum Fano resonance [27]. Additional enhancement of linear absorption and $\mathrm{TH}$ radiation can be observed at the excitation wavelengths of intrinsically 


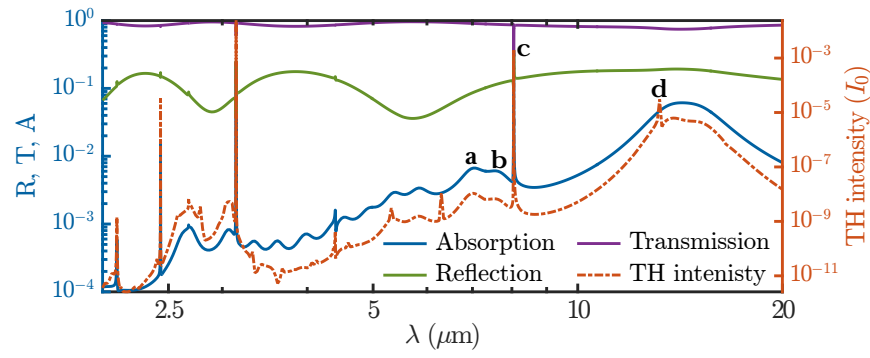

Fig. 5. Spectrum of linear absorption, reflection, and transmission and nonlinear radiation by a slab waveguide covered with graphene ribbons.

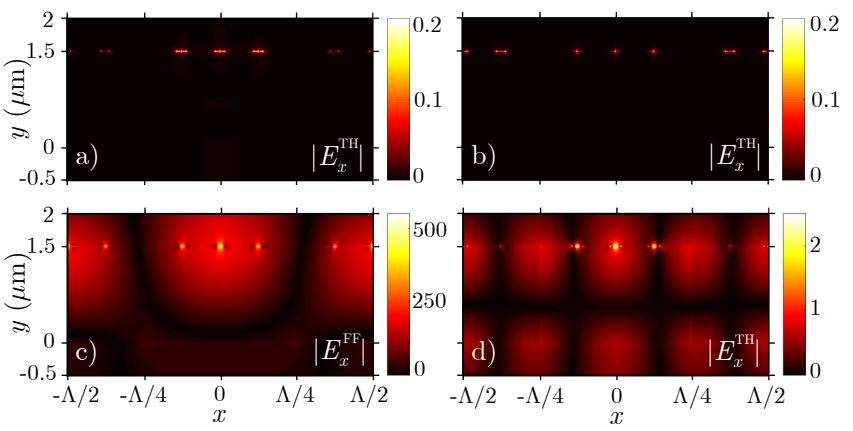

Fig. 6. a,b) TH electric near-field at surface plasmon resonance wavelength around inner (a) and outer (b) graphene ribbons. c) Fundamental electric near-field at excitation wavelength of $\mathrm{TM}_{0}$ mode. d) $\mathrm{TH}$ electric near-field at the excitation wavelength of the nonlinear $\mathrm{TM}_{0}$ mode.

nonlinear modes of the waveguide, e.g. at $\lambda_{\mathrm{TH}}=4.4 \mu \mathrm{m}$ in Fig. 5.

\section{CONCLUSiOnS}

We have introduced a versatile numerical method to investigate the physical characteristics of nonlinear optical interactions in nanopatterned 2D materials and identified different mechanisms by which these nonlinear interactions can be enhanced. Due to its general formulation, it can be applied to more complex structures that incorporate in one device graphene and other 2D materials with markedly different nonlinear optical response.

\section{REFERENCES}

[1] K. S. Novoselov, A. K. Geim, S. Morozov, D. Jiang, Y. Zhang, S. Dubonos, , I. Grigorieva, and A. Firsov, "Electric field effect in atomically thin carbon films," Science, vol. 306, no. 5696, pp. 666$669,2004$.

[2] C. Lee, X. Wei, J. W. Kysar, and J. Hone, "Measurement of the elastic properties and intrinsic strength of monolayer graphene," Science, vol. 321, no. 5887, pp. 385-388, 2008.

[3] I. Frank, D. M. Tanenbaum, A. Van der Zande, and P. L. McEuen, "Mechanical properties of suspended graphene sheets," J. Vac. Sci. Technol. B, vol. 25, no. 6, pp. 2558-2561, 2007.

[4] J. C. Meyer, A. K. Geim, M. Katsnelson, K. Novoselov, T. Booth, and S. Roth, "The structure of suspended graphene sheets," Nature, vol. 446, no. 7131, pp. 60-63, 2007.

[5] A. A. Balandin, S. Ghosh, W. Bao, I. Calizo, D. Teweldebrhan, F. Miao, and C. N. Lau, "Superior thermal conductivity of single-layer graphene," Nano Lett., vol. 8, no. 3, pp. 902-907, 2008.

[6] S. Ghosh, I. Calizo, D. Teweldebrhan, E. Pokatilov, D. Nika, A. Balandin, W. Bao, F. Miao, and C. N. Lau, "Extremely high thermal conductivity of graphene: Prospects for thermal management applications in nanoelectronic circuits," Appl. Phys. Lett., vol. 92, no. 15, p. $151911,2008$.
[7] S. Morozov, K. Novoselov, M. Katsnelson, F. Schedin, D. Elias, J. Jaszczak, and A. Geim, "Giant intrinsic carrier mobilities in graphene and its bilayer," Phys. Rev. Lett., vol. 100, no. 1, p. 016602 , 2008

[8] Y.-M. Lin, C. Dimitrakopoulos, K. A. Jenkins, D. B. Farmer, H.-Y. Chiu, A. Grill, and P. Avouris, "100-ghz transistors from wafer-scale epitaxial graphene,” Science, vol. 327, no. 5966, pp. 662-662, 2010.

[9] M. Xu, T. Liang, M. Shi, and H. Chen, "Graphene-like twodimensional materials," Chem. Rev., vol. 113, no. 5, pp. 3766-3798, 2013.

[10] Q. H. Wang, K. Kalantar-Zadeh, A. Kis, J. N. Coleman, and M. S. Strano, "Electronics and optoelectronics of two-dimensional transition metal dichalcogenides," Nature Nanotechnol., vol. 7, no. 11, pp. 699712, 2012.

[11] R. Nair, P. Blake, A. Grigorenko, K. Novoselov, T. Booth, T. Stauber, N. Peres, and A. Geim, "Fine structure constant defines visual transparency of graphene," Science, vol. 320, no. 5881, pp. 1308-1308, 2008.

[12] F. Bonaccorso, Z. Sun, T. Hasan, and A. Ferrari, "Graphene photonics and optoelectronics," Nature Photon., vol. 4, no. 9, pp. 611-622, 2010.

[13] B. Radisavljevic, A. Radenovic, J. Brivio, V. Giacometti, and A. Kis, "Single-layer mos2 transistors," Nature Nanotechnol., vol. 6, no. 3, pp. 147-150, 2011.

[14] X. Li, W. Cai, J. An, S. Kim, J. Nah, D. Yang, R. Piner, A. Velamakanni, I. Jung, E. Tutuc, S. K. Banerjee, L. Colombo, and R. S. Ruoff, "Large-area synthesis of high-quality and uniform graphene films on copper foils," Science, vol. 324, no. 5932, pp. 1312-1314, 2009.

[15] M. Weismann and N. C. Panoiu, "Theoretical and computational analysis of second- and third-harmonic generation in periodically patterned 2d-3d heteromaterials," Phys. Rev. B , in preparation 2016.

[16] A. Taflove and S. C. Hagness, Computational Electrodynamics. Artech House, 2005.

[17] J. M. Jin, The Finite Element Method in Electromagnetics. John Wiley \& Sons, 2014.

[18] A. Y. Nikitin, F. Guinea, F. J. Garcia-Vidal, and L. Martin-Moreno, "Surface plasmon enhanced absorption and suppressed transmission in periodic arrays of graphene ribbons," Phys. Rev. B, vol. 85, p. 081405 , Feb 2012.

[19] V. Nayyeri, M. Soleimani, and O. M. Ramahi, "Modeling graphene in the finite-difference time-domain method using a surface boundary condition," IEEE Trans. Antennas Propag., vol. 61, no. 8, pp. 41764182, 2013.

[20] F. H. Koppens, D. E. Chang, and F. J. Garcia de Abajo, "Graphene plasmonics: a platform for strong light-matter interactions," Nano Lett., vol. 11, no. 8, pp. 3370-3377, 2011.

[21] S.-Y. Hong, J. I. Dadap, N. Petrone, P.-C. Yeh, J. Hone, and R. M. Osgood Jr, "Optical third-harmonic generation in graphene," Phys. Rev. $X$, vol. 3, no. 2, p. $021014,2013$.

[22] J. L. Cheng, N. Vermeulen, and J. E. Sipe, "Third order optical nonlinearity of graphene," New J. Phys., vol. 16, p. 053014, 2014.

[23] M. G. Moharam, T. K. Gaylord, E. B. Grann, and D. A. Pommet, "Formulation for stable and efficient implementation of the rigorous coupled-wave analysis of binary gratings," J. Opt. Soc. Am. A., vol. 12, no. 5, pp. 1068-1076, May 1995.

[24] L. Li, "New formulation of the Fourier modal method for crossed surface-relief gratings," J. Opt. Soc. Am. A., vol. 14, pp. 2758-2767, 1997.

[25] Y. Li, A. Chernikov, X. Zhang, A. Rigosi, H. M. Hill, A. M. van der Zande, D. A. Chenet, E.-M. Shih, J. Hone, and T. F. Heinz, "Measurement of the optical dielectric function of monolayer transition-metal dichalcogenides: $\mathrm{MoS}_{2}, \mathrm{MoSe}_{2}, \mathrm{WS}_{2}$, and $\mathrm{WSe}_{2}, "$ Phys. Rev. B, vol. 90, p. 205422, Nov 2014.

[26] C. Janisch, Y. Wang, D. Ma, N. Mehta, A. L. Elas, N. Perea-Lpez, M. Terrones, V. Crespi, and Z. Liu, "Extraordinary second harmonic generation in tungsten disulfide monolayers," Sci. Rep., vol. 4, p. 5530, Jul 2014.

[27] E. J. Osley, C. G. Biris, P. G. Thompson, R. R. F. Jahromi, P. A. Warburton, and N. C. Panoiu, "Fano resonance resulting from a tunable interaction between molecular vibrational modes and a double continuum of a plasmonic metamolecule," Phys. Rev. Lett. , vol. 110 , p. 087402 , Feb 2013. 